\section{Drs. Nakabo and Ohmura reply}

\section{To the Editor:}

We appreciate the comments of Drs. Mahler and Fritzler ${ }^{1}$ in the letter to the editor regarding our article ${ }^{2}$. We will sincerely reply to the concerns raised by the authors.

We acknowledge that interlaboratory differences in anticarbamylated protein (anti-CarP) ELISA are an important issue. Although we slightly modified the original method used by Shi, et $a l^{3}$, the prevalence of anti-CarP antibodies in rheumatoid arthritis (RA) using our method was similar to that reported previously ${ }^{4}$. Therefore, we consider our in-house ELISA to be equivalent to those used in other laboratories. Our recent study gives a detailed protocol of our system ${ }^{5}$.

We agree with the comment regarding the relationship between pre-test probability and sensitivity/specificity. We would like to correct the sentence "although the pre-test probability deeply affects the sensitivity and specificity of anti-CarP antibodies in daily clinical practice, our data suggest that antiCarP antibodies cannot be used for differentiating [anticitrullinated protein antibodies] ACPA-negative RA from non-RA [connective tissue disease] CTD" in the Discussion section to "although sensitivity and specificity differ depending on comparators, our results suggest that anti-CarP antibodies cannot be used to differentiate ACPA-negative RA from non-RA CTD."

According to the suggestions provided, we prepared Venn diagrams showing the number of patients testing positive for each antibody (Figure 1). Triple-positive patients were mainly observed in the RA group. However, the OR of ACPA, rheumatoid factor (RF), and anti-CarP antibodies for the diagnosis of RA in our cohort were 57.3 (95\% CI 35.4-92.9), 14.3 (9.4-21.9), and 2.8 (2.1-3.9), respectively. In the group with ACPA and RF, OR was elevated to 84.3 (48.9-145.4), whereas it was as low as 55.1 (23.8-128.0) in the triple-positive group. The addition of the anti-CarP test to ACPA and RF did not increase OR, at least not in our cohort.

We agree with Drs. Mahler and Fritzler that our cohort does not reflect daily clinical settings, and the combined test of ACPA, RF, and anti-CarP antibodies may contribute to a preclinical diagnosis and very early intervention; however, when we want to exclude the possibility of CTD, our cohort is appropriate for testing the utility of the anti-CarP antibody. The careful exclusion of non-RA CTD is required in any cohort.

Regarding ELISA, using 1 specific protein or peptide as an antigen, we previously reported that albumin is one of the target antigens of anti-CarP antibodies ${ }^{5}$, and the prevalence of anti-CarP albumin antibodies in each CTD was similar to that of anti-CarP antibodies (Figure 2). Previous studies reported that antibodies against carbamylated fibrinogen ${ }^{6}$ and vimen- tin-derived peptide ${ }^{7}$ coexisted with ACPA. Although we agree that the combination of these specific ELISA may contribute to the diagnosis of RA, the clinical efficacy of these antibodies in seronegative RA may be limited.

SHUICHIRO NAKABO, MD, PhD, physician; KOICHIRO OHMURA, MD, $\mathrm{PhD}$, associate professor, Department of Rheumatology and Clinical Immunology, Graduate School of Medicine, Kyoto University, Kyoto, Japan. Address correspondence to Dr. K. Ohmura, Department of Rheumatology and Clinical Immunology, Graduate School of Medicine Kyoto University, 54 Shogoin-Kawahara-cho, Sakyo-ku, Kyoto, Japan. E-mail: ohmurako@kuhp.kyoto-u.ac.jp

\section{REFERENCES}

1. Mahler M, Fritzler MJ. Diagnostic utility of anticarbamylated protein antibodies as measured using carbamylated fetal calf serum. J Rheumatol 2018;45:438-9.

2. Nakabo S, Yoshifuji H, Hashimoto M, Imura Y, Nakashima R, Murakami K, et al. Anti-carbamylated protein antibodies are detectable in various connective tissue diseases. J Rheumatol 2017;44:1384-8.

3. Shi J, Knevel R, Suwannalai P, van der Linden MP, Janssen GM, van Veelen PA, et al. Autoantibodies recognizing carbamylated proteins are present in sera of patients with rheumatoid arthritis and predict joint damage. Proc Natl Acad Sci U S A 2011;108:17372-7.

4. Li L, Deng C, Chen S, Zhang S, Wu Z, Hu C, et al. Meta-analysis: diagnostic accuracy of anti-carbamylated protein antibody for rheumatoid arthritis. PLoS One 2016;11:e0159000.

5. Nakabo S, Hashimoto M, Ito S, Furu M, Ito H, Fujii T, et al Carbamylated albumin is one of the target antigens of anti-carbamylated protein antibodies. Rheumatology 2017; 56:1217-26.

6. Scinocca M, Bell DA, Racape M, Joseph R, Shaw G, McCormick $\mathrm{JK}$, et al. Antihomocitrullinated fibrinogen antibodies are specific to rheumatoid arthritis and frequently bind citrullinated proteins/peptides. J Rheumatol 2014;41:270-9.

7. Juarez M, Bang H, Hammar F, Reimer U, Dyke B, Sahbudin I, et al. Identification of novel antiacetylated vimentin antibodies in patients with early inflammatory arthritis. Ann Rheum Dis 2016; 75:1099-107.

J Rheumatol 2018;45:3; doi:10.3899/jrheum.171415

B

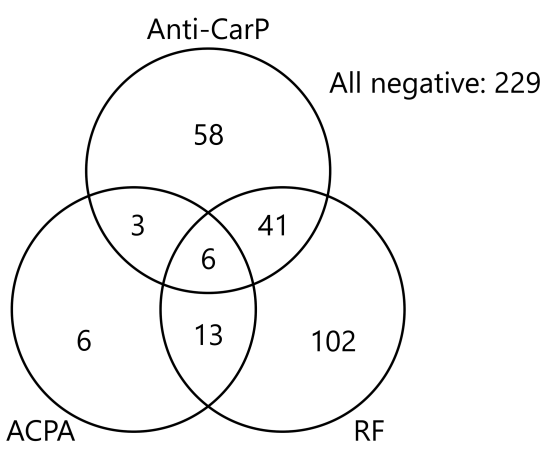

non-RA $\operatorname{CTD}(\mathrm{n}=458)$

$$
\text { RA }(n=265)
$$

All negative: 27

Figure 1. Venn diagrams of ACPA, anti-CarP antibodies, and RF in RA (A) and non-RA CTD (B). ACPA: anticitrullinated peptide antibodies; anti-CarP: anticarbamylated protein antibodies; RF: rheumatoid factor; RA: rheumatoid arthritis; CTD: connective tissue diseases. 


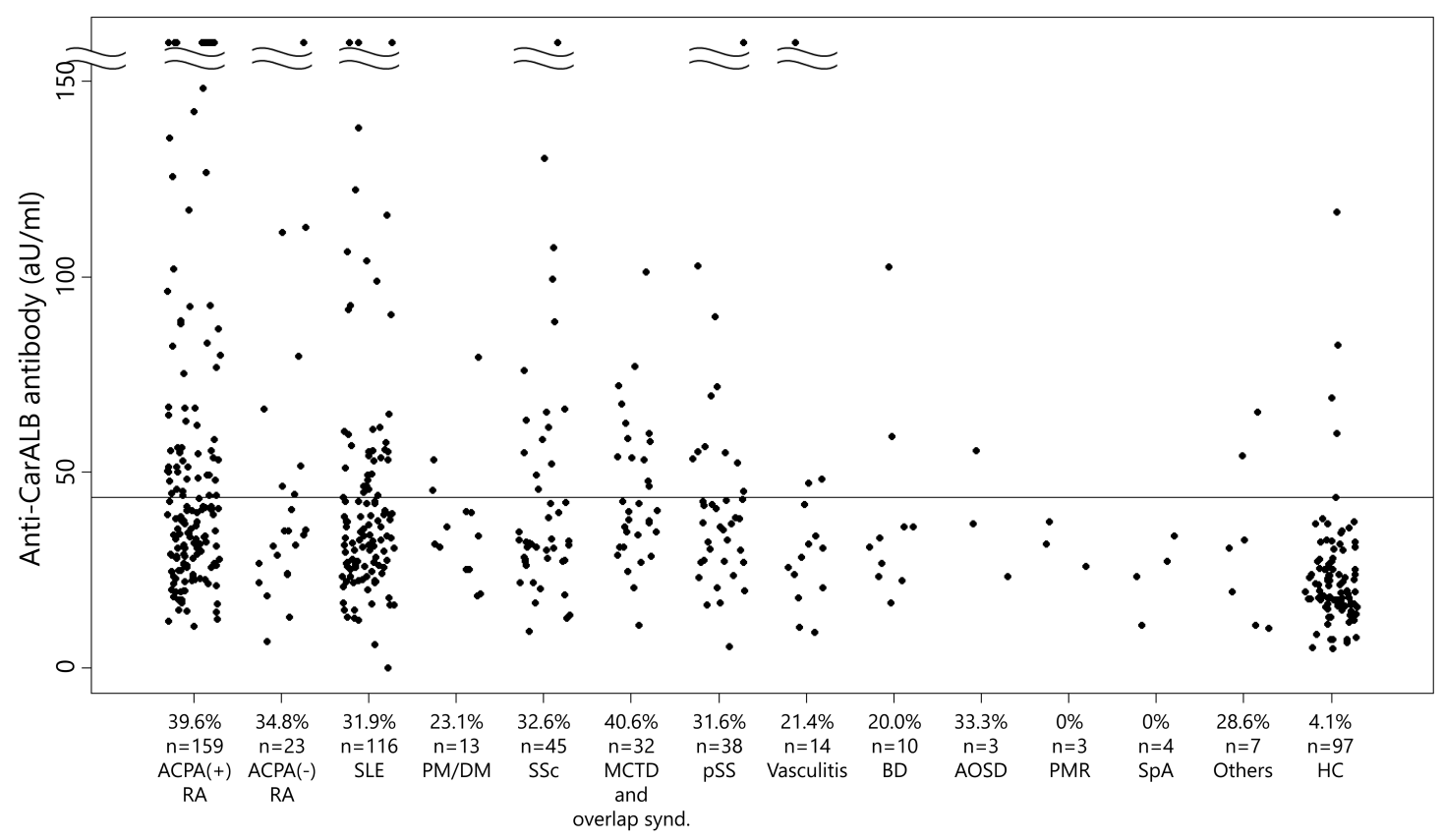

Figure 2. Anti-CarALB antibody prevalence and levels in RA and other CTD. The horizontal line represents the cutoff value. The anti-CarALB level was measured and the cutoff value was selected as described in our previous study ${ }^{5}$. Anti-CarALB: anticarbamylated albumin; RA: rheumatoid arthritis; CTD: connective tissue diseases; ACPA: anticitrullinated peptide antibodies; SLE: systemic lupus erythematosus; PM/DM: polymyositis/dermatomyositis; SSc: systemic sclerosis; MCTD: mixed connective tissue diseases; pSS: primary Sjögren syndrome; BD: Behçet disease; AOSD: adult-onset Still disease; PMR: polymyalgia rheumatica; SpA: spondyloarthritis; HC: healthy controls. 